\title{
Clinicopathological features of serrated adenoma of the colorectum: comparison with traditional adenoma
}

\author{
Takayuki Matsumoto, Mitsuru Mizuno, Michio Shimizu, Toshiaki Manabe, Mitsuo Iida
}

\begin{abstract}
Aim-Serrated adenoma is a recently described category of colorectal adenoma. The aim of this study was to determine whether serrated adenomas have clinicopathological findings distinct from "traditional" adenoma.

Methods-Colonoscopy records for 19951998 were reviewed, and all traditional adenomas and serrated adenomas identified. Tumour site and endoscopic configuration were compared between the two types of adenoma. In polypectomised lesions, size and grade of dysplasia were compared between the two groups.

Results-There were 3305 traditional adenomas and 43 serrated adenomas. Serrated adenomas were more often found in the rectosigmoid colon than the traditional adenomas $(67.5 \% \quad v \quad 41.2 \%$, $\mathrm{p}=0.0008)$. Polypectomy was more often done for serrated adenomas (27 of 43 lesions, $62.8 \%$ ) than for traditional adenomas (626 of 3305 lesions, $18.9 \%$, $\mathrm{p}<0.0001$ ). Size (mean (SD) maximum dimension, 12 (6) $\mathrm{mm} v 13(3) \mathrm{mm}$ ) and incidence of high grade dysplasia $(14.8 \% v$ $13.6 \%$ ) in endoscopically removed specimens were not different between the two types.

Conclusions-The therapeutic judgement applied to the management of traditional adenoma seems to be appropriate for serrated adenoma.

(F Clin Pathol 1999;52:513-516)
\end{abstract}

Keywords: colon; adenoma; serrated adenoma; colonoscopy

Hyperplastic (metaplastic) polyps of the colorectum have been regarded as benign, nonneoplastic protrusions. Discussion of their clinical significance has mainly focused on whether the presence of this type of lesion is

Accepted for publicatio 4 March 1999

Table 1 Comparison of clinical features between subjects with and without serrated adenoma

\begin{tabular}{lll}
\hline & $\begin{array}{l}\text { Subjects without serrated } \\
\text { adenoma }(n=1438)\end{array}$ & $\begin{array}{l}\text { Subjects with serrated } \\
\text { adenoma }(n=36)\end{array}$ \\
\hline $\begin{array}{l}\text { Age, years (mean (SD)) } \\
\text { Sex (female/male) }\end{array}$ & $62.3(10.8)$ & $63.1(11.3)$ \\
$\begin{array}{l}\text { Synchronous lesion } \\
\text { Traditional adenoma }\end{array}$ & $415 / 1023$ & $10 / 26$ \\
$\quad \begin{array}{l}\text { Invasive cancer } \\
\text { Number of adenomas }\end{array}$ & $1438(100 \%)$ & $21(58 \%)$ \\
$\quad<4$ & $85(6 \%)$ & $0(0 \%)$ \\
$4-6$ & $1206(84 \%)$ & $26(72 \%)$ \\
$>6$ & $175(12 \%)$ & $8(22 \%)$ \\
\hline
\end{tabular}

*Total number of traditional and serrated adenomas. predictive of the simultaneous or metachronous occurrence of colorectal neoplasia. ${ }^{1-4}$ There have been reports, however, of cases of hyperplastic polyps associated with adenomatous or cancerous areas. ${ }^{5-8}$

In 1990, Longacre and Fenoglio-Preiser ${ }^{9}$ described a new category of colorectal neoplasia with a serrated glandular configuration, and pathologists now refer to such lesions as "serrated adenomas." While there has been a subsequent description of the histopathological features of serrated adenoma, ${ }^{10}$ the clinical significance and treatment implications of these lesions have not been examined. In this study, we investigated whether serrated adenomas have clinicopathological features distinct from the "traditional" type of adenoma, so that it might be possible to establish a therapeutic strategy for this pathologically unique but still insufficiently recognised neoplasm.

\section{Methods}

We retrospectively investigated all the colonoscopy records performed for a total of 3575 subjects at our institution during the period from February 1995 until January 1998. We excluded subjects in whom total colonoscopy could not be performed and those with the established diagnosis of familial adenomatous polyposis or chronic inflammatory bowel disease. We assessed all endoscopically and histologically verified neoplasms in the remaining subjects.

Histological slides of all the biopsy or polypectomy specimens obtained from these colorectal neoplasms were reviewed by two pathologists (Shimizu and Manabe), and epithelial neoplasms limited to the mucosa were classified into either traditional adenomas or serrated adenomas. The histological diagnosis of traditional adenoma was based upon the widely accepted criteria for epithelial tumours of the intestine. ${ }^{11}$ The diagnosis of serrated adenoma was made according to the criteria of Longacre and Fenoglio-Preiser. ${ }^{9}$ In brief, a neoplasm with a serrated glandular pattern simulating hyperplasia, and composed of cells with prominent nuclei, was regarded as a serrated adenoma. Adenomas with distinctive hyperplastic areas, which have been referred to as mixed hyperplastic-adenomatous polyps, ${ }^{8}$ were categorised as traditional adenomas.

We determined the grade of dysplasia in both traditional and serrated adenomas that were removed either by polypectomy or by endoscopic mucosal resection. Because of recent criticism that Japanese pathologists tend to 


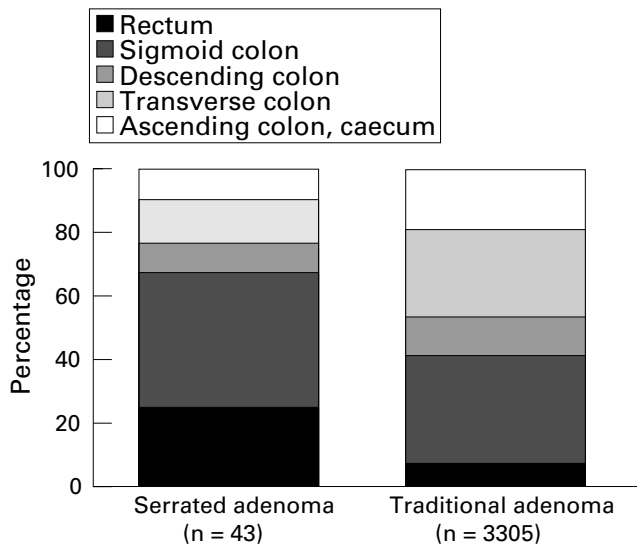

Figure 1 Comparison of site within colorectum between serrated adenoma and traditional adenoma. Serrated adenomas were more often found in the rectum or in the sigmoid colon than traditional adenomas.

Table 2 Comparison of endoscopic appearances between traditional and serrated adenomas

\begin{tabular}{lll}
\hline & $\begin{array}{l}\text { Traditional adenoma } \\
(n=3305)\end{array}$ & $\begin{array}{l}\text { Serrated adenoma } \\
(n=43)\end{array}$ \\
\hline Pedunculated & $384(11.6)$ & $10(23.3)$ \\
Semipedunculated & $1615(48.9)$ & $20(46.5)$ \\
Sessile & $1235(37.4)$ & $12(27.9)$ \\
Carpet-like & $51(1.5)$ & $1(2.3)$ \\
Non-polypoid & $20(0.6)$ & $0(0.0)$ \\
\hline
\end{tabular}

Values in parentheses are percentages among each type of adenoma.

overestimate the degree of dysplasia, ${ }^{12}$ an initial diagnosis of carcinoma in situ was redefined as high grade dysplasia, and the rest as low grade dysplasia, in order to avoid misinterpreting the data.

In this pathological review we identified 1474 subjects with traditional adenoma, serrated adenoma, or both. The sex, the age at colonoscopy, and presence of other colorectal neoplasms were compared between subjects with and without serrated adenoma.

We then compared the site within the colorectum, the endoscopic appearances, and the treatment of each type of adenoma. The endoscopic appearance was classified into pedunculated, semipedunculated, sessile, carpet-like, or non-polypoid. "Non-polypoid" configuration was defined when the lesion had a flat topped elevation with a central depression, as previously described. ${ }^{13} 14$ In addition, we also compared the grade of dysplasia in those adenomas that were completely removed by polypectomy or endoscopic mucosal resection.

\section{STATISTICS}

The data are expressed as mean (SD). When comparing between the two groups, we used the $\chi^{2}$ test, Fisher's exact probability test, or Student's $t$ test. Probabilities of less than 0.05 were considered to be significant.

\section{Results}

We identified 3348 adenomas in 1474 subjects. These adenomas were divided into 3305 traditional adenomas (including one mixed hyperplastic-adenomatous polyp) and 43 serrated adenomas. Thus the serrated adenomas comprised $1.3 \%$ of all the adenomas found during the investigation period. There were no lesions over which the two pathologists were in disagreement. There were 36 subjects with serrated adenomas and 1438 without. Of the subjects with serrated adenomas, 32 had a single adenoma, and four had multiple adenomas; two of the latter had two adenomas, and the other two had three and four, respectively.
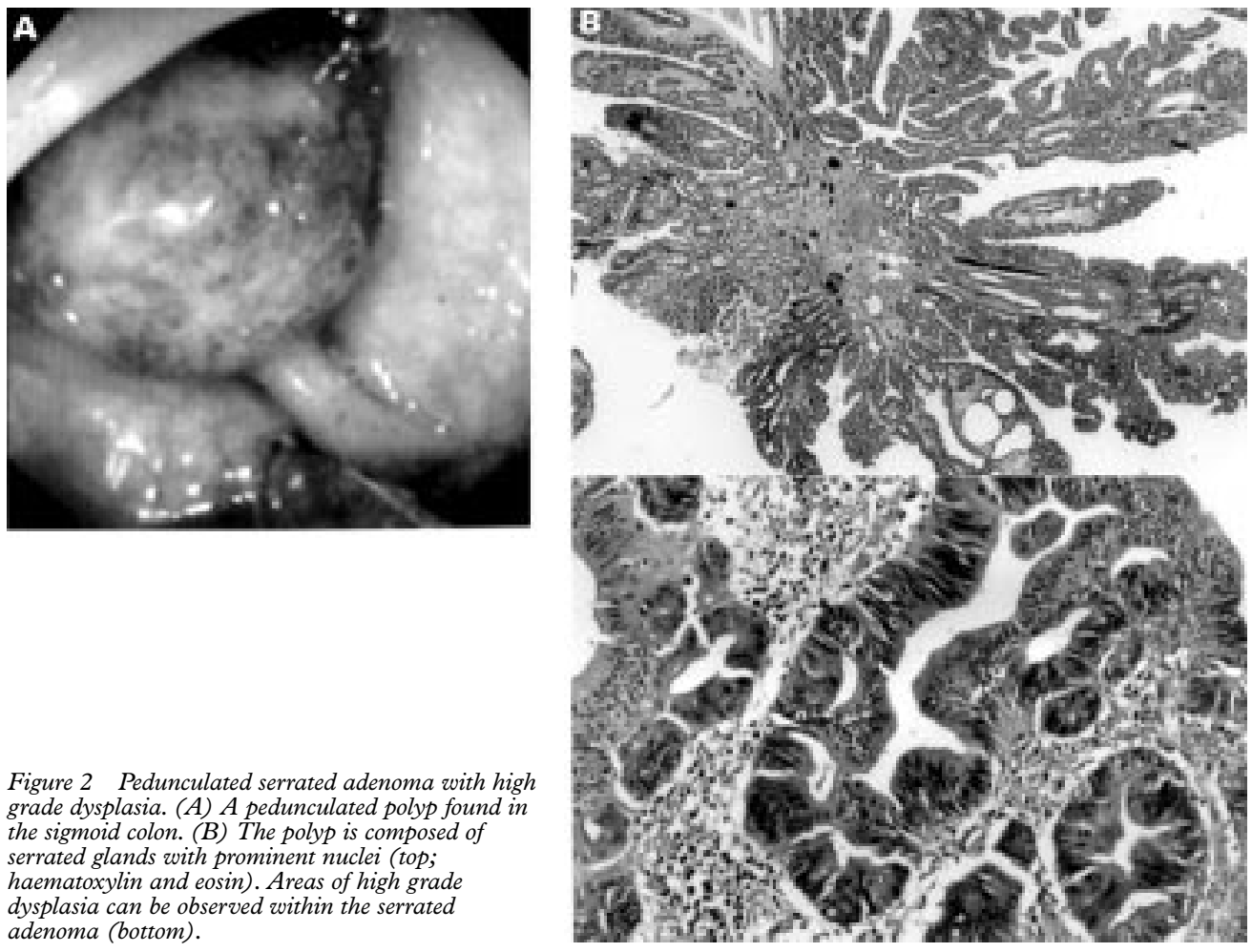

Figure 2 Pedunculated serrated adenoma with high grade dysplasia. (A) A pedunculated polyp found in the sigmoid colon. (B) The polyp is composed of serrated glands with prominent nuclei (top; haematoxylin and eosin). Areas of high grade dysplasia can be observed within the serrated adenoma (bottom). 

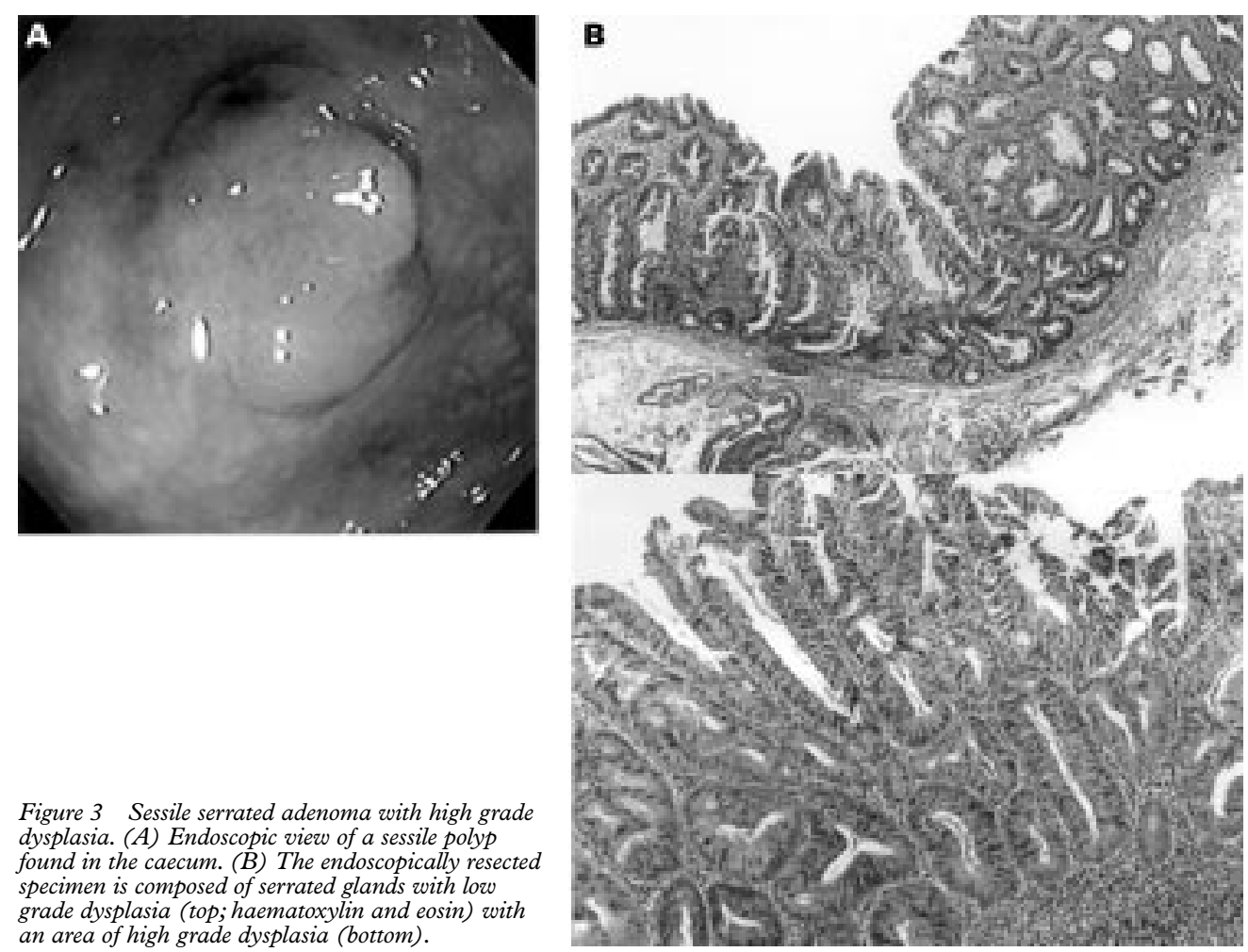

Figure 3 Sessile serrated adenoma with high grade dysplasia. (A) Endoscopic view of a sessile polyp found in the caecum. (B) The endoscopically resected specimen is composed of serrated glands with low grade dysplasia (top; haematoxylin and eosin) with an area of high grade dysplasia (bottom).

COMPARISON OF CLINICAL FEATURES

A comparison of the clinical features of subjects with and without serrated adenomas is given in table 1. Age and sex were not significantly different between the two groups of subjects. While 85 of the 1438 subjects without serrated adenomas had coexisting cancers with submucosal invasion, none of the subjects with serrated adenomas had invasive cancer. This difference in the incidence of invasive cancer did not, however, reach significance $(p=0.114)$. The average number of adenomas was not different between subjects with serrated adenomas and those without, at 2.3 (1.9) v 2.6 (2.0).

\section{COMPARISON OF SITE AND CONFIGURATION}

Figure 1 gives a comparison of the site of origin of the tumours within the colorectum. Twenty nine of the 43 serrated adenomas were located in the rectum or sigmoid colon, while 1355 of the 3305 traditional adenomas were found at these sites $(67.5 \% v 41.0 \%, \mathrm{p}=0.0008)$.

A comparison of endoscopic appearance is given in table 2. Polypoid configuration was predominant in both traditional adenomas and serrated adenomas. While a non-polypoid configuration was noted in 20 traditional adenomas, such a configuration did not occur with serrated adenomas. However, there was no statistical difference in endoscopic configuration between the two types of adenoma.

COMPARISON OF TREATMENT AND THE GRADE OF DYSPLASIA

Endoscopic polypectomy or endoscopic mucosal resection was done in 626 traditional adenomas and 27 serrated adenomas, while in the remaining lesions endoscopic removal was not thought to be indicated. Endoscopic removal was more often done for serrated adenomas $(62.8 \%)$ than for traditional adenomas $(18.9 \%)(p<0.0001)$

The size of the polypectomised specimens did not differ significantly between serrated adenomas and traditional adenomas, at 12 (6) $\mathrm{mm} v 13$ (3) $\mathrm{mm}$. There were four serrated adenomas which contained areas of high grade dysplasia (figs 2 and 3). The incidence of high grade dysplasia, however, was not statistically different between serrated adenomas (14.8\%) and traditional adenomas (85 of 626 adenomas, $13.6 \%$ ).

\section{Discussion}

The presence of neoplastic change within hyperplastic polyps or in neoplasms mimicking hyperplasia in the general population, ${ }^{6-9}$ in metaplastic polyposis, ${ }^{15}{ }^{16}$ or even in hereditary non-polyposis colorectal cancer ${ }^{5}$ has raised the possibility that hyperplastic polyps may be another precursor of human colorectal cancer. The serrated adenoma can be regarded as a specific lesion which might explain the occurrence of neoplasia within hyperplastic polyps. Our results indicate that serrated adenomas were much less common than traditional adenomas. However, the incidence of serrated adenoma could have been underestimated, because for the current investigation we excluded diminutive polyps suggestive of hyperplastic polyps.

Since the initial report by Longacre and Fenoglio-Preiser, ${ }^{9}$ serrated adenoma has mainly been discussed in relation to its pathological or immunohistochemical features. ${ }^{10} 17$ Rubio and Rodensjö made an extensive analysis of small serrated adenomas and emphasised that these tumours may be another precursor of invasive colorectal cancer, because a 
proportion of them contained areas of high grade dysplasia or even invasive cancer. ${ }^{18}$ Furthermore, since a recent colonoscopic survey by the same investigators showed that small serrated adenomas had endoscopic or histological features similar to hyperplastic polyps, ${ }^{19}$ it seems necessary to establish a therapeutic strategy for these apparently indolent lesions.

Our result indicate that a polypoid configuration is predominant in both serrated adenomas and traditional adenomas, and a nonpolypoid configuration was not identified in the former group. In contrast, Jaramillo et al reported that 29 of their 54 serrated adenomas showed a "flat" configuration. ${ }^{19}$ The different prevalence of non-polypoid serrated adenoma in our sample and in that of Jaramillo et al may be related to differences in defining the endoscopic appearances; thus we defined a flat elevation without any depression as a sessile configuration. Our results showing that serrated adenomas were more often treated by endoscopic polypectomy suggest that this type of adenoma has a rather more exophytic growth pattern than the traditional adenoma.

In our colonoscopic survey, serrated adenomas were found more often in the distal part of the colorectum than traditional adenomas. This is in contrast with the description given by Longacre and Fenoglio-Preiser, ${ }^{9}$ who did not find any difference in site specificity between serrated adenoma and traditional adenoma. This may be explained by differences in the method of data collection. An endoscopic survey by Jaramillo et al also showed that $78 \%$ of their serrated adenomas were found in the rectosigmoid colon, ${ }^{19}$ so a predominantly distal location seems to be one of the clinicopathological features specific to this type of adenoma. Our results also seem to confirm that a certain proportion of hyperplastic polyps, which predominantly develop in the distal part of the colorectum, transform into neoplasms. ${ }^{20}$

As the age, sex, and the number of coexisting colorectal neoplasms were not obviously different between subjects with and without serrated adenoma, and because more than half of the former had an accompanying traditional adenoma, we speculate that both serrated adenoma and traditional adenoma share common predisposing factors in their pathogenesis. While it has been shown that the serrated adenoma has a different growth pattern from the traditional adenoma with respect to proliferative activity ${ }^{10}{ }^{17}$ and $\mathrm{p} 53$ overexpression, ${ }^{18}$ the clinical implications of serrated adenomas for the development of invasive cancer remain to be established. The results of our study suggest that any therapeutic considerations relevant to traditional adenoma should also be applied to serrated adenoma, because if they were of equivalent size both types of adenoma had a similar incidence of high grade dysplasia. As this study was based on a retrospective analysis, further studies of metachronous lesions in subjects with serrated adenomas are necessary to establish the clinical significance of this type of adenoma.

1 Croizet O, Moreau J, Arany Y, et al. Follow-up of patients with hyperplastic polyps of the large bowel. Gastrointest Endosc 1997;46:119-23.

2 Sciallero S, Cosantini M, Bertinelli E, et al. Distal Sciallero S, Cosantini $\mathrm{M}$, Bertinelli $\mathrm{E}$, et al. Distal
hyperplastic polyps do not predict proximal adenomas. hyperplastic polyps do not predict proximal adenomas.
Results from a multicentric study of colorectal adenomas. Gastrointest Endosc 1997;46:124-30.

3 Provenzale D, Garrett JW, Condon SE, et al. Risk for colon adenomas in patients with rectosigmoid hyperplastic polyps. Ann Intern Med 1990;113:760-3.

4 Foutch PG, DiSario JA, Pardy K, et al. The sentinel hyperplastic polyp: a marker for synchronous neoplasia in the proximal colon. Am f Gastroenterol 1991;86:1482-5.

5 Jass JR, Cottier DS, Pokos V, et al. Mixed epithelial polyps in association with hereditary non-polyposis colorectal cancer providing an alternative pathway of cancer histogenesis. Pathology 1997;29:28-33.

6 Franzin G, Novelli P. Adenocarcinoma occurring in a hyperplastic (metaplastic) polyp of the colon. Endoscopy 1982;14:28-30.

7 Teoh HH, Delahunt B, Isbister WH. Dysplastic and malignant areas in hyperplastic polyps of the large intestine. Pathology 1989;21:138-42.

8 Urbanski SJ, Marcon N, Kossakowska AE, et al. Mixed hyperplastic adenomatous polyps: an underdiagnosed entity. Am f Surg Pathol 1984;8:551-6.

9 Longacre TA, Fenoglio-Preiser CM. Mixed hyperplastic adenomatous polyps/serrated adenomas. A distinct form of colorectal neoplasia. Am f Surg Pathol 1990;14:524-37.

10 Kang M, Mitomi H, Sada M, et al. Ki-67, p53, and Bcl-2 expression of serrated adenomas of the colon. Am 7 Surg Pathol 1997;21:417-23.

11 Jass JR, Sobin LH. Histological typing of intestinal tumours. Geneva: World Health Organisation, 1994.

12 Schlemper RJ, Itabashi M, Kato Y, et al. Differences in diagnostic criteria for gastric carcinoma between Japanese and Western pathologists. Lancet 1997;349:1725-9.

13 Matsumoto T, Iida M, Yao T, et al. Minute non-polypoid adenoma of the colorectum. Correlation between endoscopic and histologic findings. Gastrointest Endosc 1992;38: 645-50.

14 Matsumoto T, Iida M, Yao T, et al. Role of nonpolypoid neoplastic lesions in the pathogenesis of colorectal cancer. neoplastic lesions in the pathogen
Dis Colon Rectum 1994;37:450-5.

15 McCann BG. A case of metaplastic polyposis of the colon ssociated with focal adenomatous change and metachronous adenocarcinomas. Histopathology 1988;13:700-3.

16 Bengoechea O, Martínez-Peñuela JM, Larrínaga B, et al. Hyperplastic polyposis of the colorectum and adenocarcinoma in a 24-year-old man. Am F Surg Pathol 1987;11:323-

7 Fujishima N. Proliferative activity of mixed hyperplastic adenomatous polyp/serrated adenoma in the large intestine, measured by PCNA (proliferating cell nuclear antigen). F Gastroenterol 1996;31:207-13.

18 Rubio CA, Rodensjö M. p53 over expression in flat serrated adenomas and flat tubular adenomas. 7 Cancer Res Clin Oncol 1995;121:571-6.

19 Jaramillo E, Watanabe M, Rubio C, et al. Small colorectal serrated adenomas: endoscopic findings. Endoscopy 1996; 28:1-3.

20 Otori K, Oda Y, Sugiyama K, et al. High frequency of K-ras mutations in human colorectal hyperplastic polyps. Gut 1997;40:660-3. 